\title{
Perceived happiness level influences evocation of positive emotions
}

\author{
${\text { Masahiro Matsunaga }{ }^{1^{*}}, \text { Hiroki Murakami }^{2}, \text { Kaori Yamakawa }^{3} \text {, Tokiko Isowa }}^{4}$, \\ Seisuke Fukuyama ${ }^{5}$, Jun Shinoda ${ }^{5}$, Jitsuhiro Yamada ${ }^{5}$, Hideki Ohira ${ }^{3}$
}

\footnotetext{
${ }^{1}$ Division of Cerebral Integration, Department of Cerebral Research, National Institute for Physiological Sciences, Aichi, Japan;

*Corresponding Author: mmatsu@nips.ac.jp

${ }^{2}$ Department of Psychology, Stony Brook University, New York, USA;

${ }^{3}$ Department of Psychology, Graduate School of Environmental Studies, Nagoya University, Aichi, Japan;

${ }^{4}$ Faculty of Nursing, Graduate School of Medicine, Mie University, Mie, Japan;

${ }^{5}$ Chubu Medical Center for Prolonged Traumatic Brain Dysfunction, Kizawa Memorial Hospital, Gifu, Japan.
}

Received 12 April 2011; revised 30 April 2011; accepted 5 May 2011.

\begin{abstract}
Happiness is a positive feeling characterized by satisfaction, joy, pleasure, or love. Perceived happiness level is likely associated with health and well-being. However, the neurobiological mechanisms underlying the positive effects of happiness on psychological and physiological wellness remain obscure. In this study, we found that the medial prefrontal cortex (MPFC) was strongly activated and positive emotions were strongly evoked in individuals with high perceived happiness levels compared to those with low perceived happiness levels when either looked at a favorite person. Moreover, positive mood changes were positively correlated with MPFC activity. These results show that perceived happiness level is associated with positive emotion evocation by means of the MPFC function, which may be involved in psychological and physiological wellness. Therefore, happiness may play an important role in psychological and physiological wellness.
\end{abstract}

Keywords: Happiness; Medial Prefrontal Cortex; Attraction; Positron Emission Tomography

\section{INTRODUCTION}

Stress and anxiety can lead to depression [1]. Psychoneuroimmunological studies have shown that psychosocial stressors can activate the sympathetic nervous system and that prolonged elevation of sympathetic nervous activity weakens immune defense mechanisms [2]. Therefore, stress and anxiety may cause or facilitate not only depression but several other diseases as well, including cancer [3].

The idea that perceived happiness level may be associated with health and well-being has recently been explored. Individuals with a high level of perceived happiness have reduced sympathetic activation after exposure to psychological stressors compared with those having low levels of perceived happiness [4]. A recent study has also indicated that perceived happiness is negatively correlated with depression and anxiety [5]. Therefore, perceived happiness level is involved in both psychological and physiological well-being. The neurobiological mechanism that explains these positive effects of happiness on psychological and physiological well-being remains obscure. The medial prefrontal cortex (MPFC) is one of the brain regions that may be involved in psychological and physiological wellness. It has been shown that electrical stimulation of the MPFC has an antidepressant effect by increasing synaptic availability of serotonin [6], suggesting that MPFC maintains positive mood states. From this viewpoint, it is suggested that individuals with high levels of perceived happiness have stronger MPFC activity and stronger positive emotion evocation than individuals with low levels of perceived happiness upon exposure to positive emotional stimuli. To test this hypothesis, participants in this study were required to watch films featuring people that they considered attractive, and we simultaneously recorded their brain activity using ${ }^{15} \mathrm{O}$-water positron emission tomography (PET) during various mood states.

\section{METHODS}

\subsection{Participants}

We recruited 18 healthy right-handed male volunteers (age range, 20 - 29 years). None of the participants had 
chronic illness and none were taking medications known to affect immunity. Only men were included to avoid gender-related endocrine system and immune variations caused by the female menstrual cycle. To screen individuals with higher or lower levels of perceived happiness, we requested them to use the Japanese version of the subjective happiness scale (JSHS) [5]. The JSHS is a 4-item scale that measures relatively stable perceived happiness. Internal consistency, test-retest reliability, convergent validity, and discriminant validity of the JSHS have been confirmed [5]. Results of this screening investigation indicate that the mean JSHS score for the participants was 4.83 with a standard deviation (SD) of 0.9 , similar to those reported earlier [5]. According to the mean participant JSHS score, we designated participants with scores $>5.0$ as higher happiness respondents $(n=9)$ and those $<4.9$ as lower happiness respondents (n $=9$ ). The participants were age-matched. All participants signed informed consent forms prior to participating in the study in accordance with university policy, and the study was approved by the Kizawa Memorial Hospital ethics committee.

\subsection{Task Procedure}

Each participant was instructed not to eat for $2 \mathrm{~h}$ before the scanning session but was allowed nonalcoholic and caffeine-free fluids. Either an emotionally neutral film (control film) or a film featuring people that the participant considered attractive (positive film) was viewed for $4 \mathrm{~min}$ on a 15 -inch display positioned approximately $60 \mathrm{~cm}$ from the participants. PET scanning (duration, $60 \mathrm{~s}$ ) was performed during the first 2 - $3 \mathrm{~min}$ of the screening. To obtain reliable PET data, each participant was assigned 2 positive and 2 control films and 4 PET scans were obtained per participant. The control film was a TV news program that featured a newscaster that the participants did not consider particularly attractive. The participants watched the edited control and positive films for the first time at the time of the experiment. The emotional valence of the films had been previously evaluated [7]. The order of the 2 film types was randomized across participants. The detailed PET procedure was described previously [7].

\subsection{Image Acquisition by PET, Image Processing, and Analysis}

During each film-watching period, the distribution of regional cerebral blood flow ( $\mathrm{rCBF}$ ) was measured using an Advance NXi PET scanner (GE Healthcare Life Sciences, Little Chalfont, England) operating in high-sensitivity 3-dimensional mode [7]. SPM99 software [8] implemented in Matlab (version 6.1; Mathworks, Sherborn,
MA) was used for spatial preprocessing and statistical analyses as described previously [7]. To clarify significant regional changes within groups (high vs. low) during the positive condition, differences between the 2 groups were analyzed by subtracting the images of the low happiness group from the images of the high happiness group (each obtained by subtracting the control condition images from those obtained during the positive condition). The effects at each voxel were estimated using a general linear model. Voxel values for each contrast yielded a statistical parametric map of the $t$-statistic (SPM $t)$, which was subsequently transformed to a unit normal distribution (SPM z). The peak voxel value significance thresholds were set at $p<0.001$ (uncorrected) and the cluster significance thresholds were set at 15 voxels.

Further, to examine the association between brain activity reflected by the $\mathrm{rCBF}$ and positive mood, we created statistical parametric maps to identify the brain regions that were activated in synchrony with the positive mood changes using the high happiness group data. For these maps, the covariates option was selected, generating regression analyses that tested the linear relationship between $\mathrm{rCBF}$ and positive mood, yielding a $\mathrm{z}$ score at each voxel. We entered the value changes obtained in the positive mood obtained by subtracting the value before watching a film from the value after watching a film as covariates. For covariate analysis, the statistical threshold was set at $p<0.0001$ (uncorrected) for height and clusters including $>20$ contiguous voxels were reported. For ease of discussion, we refer to our findings in terms of significant "correlations," although the analysis formally involved linear regression rather than correlation assessment. After voxel-wise regression analysis, the MPFC value was extracted using SPM99, and the Pearson correlation coefficient was computed between the MPFC response and the positive mood change. Both control and positive conditions $(2 \times 9$ data points $)$ were used in both the voxel-wise regression analysis and the Pearson correlation coefficient computation.

\subsection{Statistical Analyses}

The positive mood before and after film watching was compared using 3-way analysis of variance (ANOVA) (group [high vs. low] $\times$ condition [control vs. positive] $\times$ period [before vs. after]), followed by paired $t$-tests. This index was measured twice for each condition; thus, statistical analyses were conducted among participants after each participant's average was calculated.

\section{RESULTS}

Subtraction of the low happiness from the high hap- 
piness group data revealed significant increases in the rCBF of the MPFC (Brodmann area 9; $\mathrm{x}, \mathrm{y}, \mathrm{z}=-2,58$, $30)$, dorsal striatum $(x, y, z,=-16,10,-4)$, insula (x, y, $z=-36,10,0)$, and supplementary motor area (SMA; , $\mathrm{y}, \mathrm{z}=-14,8,64)$ (Figure 1). With regard to the positive mood, the ANOVA identified significant interactions of group (high vs. low) $\times$ condition (control vs. positive) $\times$ period (before vs. after) (F $[1,32]=5.75, p<0.05$; Figure 2). Further statistical analyses indicated that watching the positive film significantly elevated the positive mood in both groups: high: $\mathrm{t}(8)=5.53, p<0.01$; low: $\mathrm{t}(8)$ $=-3.41, p<0.01$ (Figure 2). However, the positive mood score after watching the positive film was higher in the high happiness group than in the low happiness group: $\mathrm{t}(8)=3.46, p<0.01$ (Figure 2). We then analyzed the correlation between change in positive mood and $\mathrm{rCBF}$ : positive correlations were identified in several brain regions, including the MPFC $(\mathrm{x}, \mathrm{y}, \mathrm{z}=-2,56$, 38 ). The MPFC value, which was activated greatly in the high happiness group, was then extracted and the Pearson correlation coefficient between the MPFC response and the positive mood change was computed: $\mathrm{r}(18)=$ $0.51, p<0.05$ (Figure 3).

\section{DISCUSSION}

We hypothesized that the MPFC may be strongly activated in individuals with high levels of perceived happiness and that strong positive emotions may be evoked upon sight of a favorite person compared to individuals with low levels of perceived happiness. As expected, this

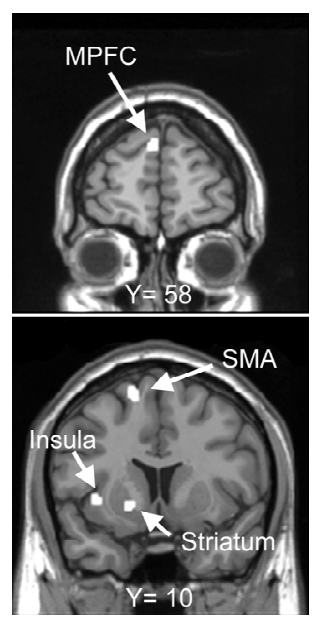

Figure 1. Brain regions activated by the positive films in the high happiness group as identified by PET and by subtracting the low happiness group values from the high happiness group values $(p<0.001$, uncorrected). PET: positron emission tomography; MPFC: medial prefrontal cortex; SMA: supplementary motor area.
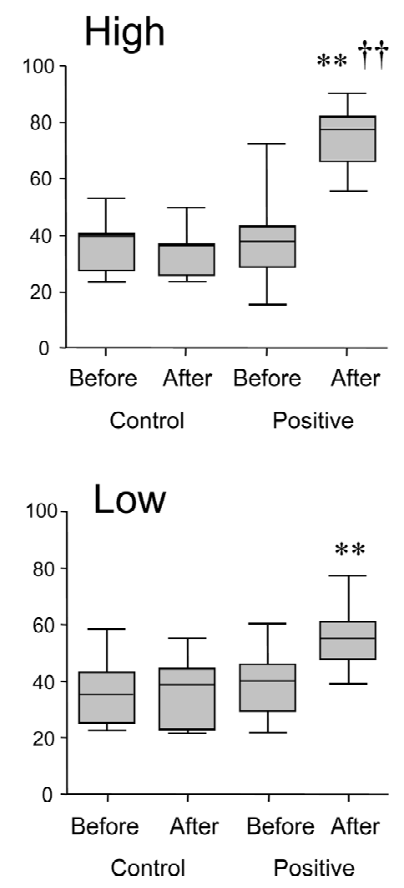

Figure 2. Change in positive mood state after watching the films. $* * p<0.01$ vs. before watching by paired $t$-test. $\dagger \uparrow p<$ 0.01 vs. after watching the positive film in the low happiness group by paired $t$-test.
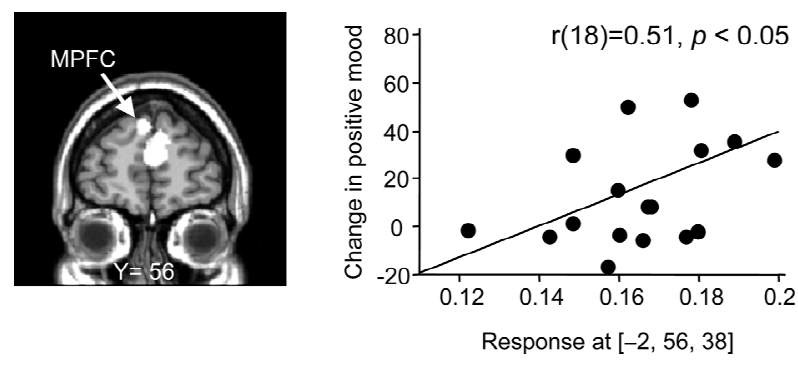

Figure 3. SPM99 covariate analysis results $(p<0.0001$, uncorrected) showing a positive correlation between change in positive mood and $\mathrm{rCBF}$ in the MPFC; scatterplot showing MPFC activity and positive mood change from baseline to after film screening $(\mathrm{r}(18)=0.51, p<0.05)$. rCBF: regional cerebral blood flow; MPFC: medial prefrontal cortex.

study showed that the high happiness group had a greater degree of MPFC activation. Moreover, strong positive emotions were evoked in participants in this group compared to participants in the low happiness group when the participants looked at a favorite person. Covariate analysis indicated that MPFC activation was positively correlated with positive mood increases. These results show that perceived happiness level is associated with positive emotion evocation via MPFC function. Thus, individuals with high levels of perceived happiness may maintain psychological well-being via MPFC function. In addition, MPFC can control auto- 
nomic nervous system activity via brain autonomic centers such as the hypothalamus and midbrain through direct neural projections [9]. Thus, it is possible that reduced sympathetic activation in individuals with high levels of perceived happiness after exposure to psychosocial stressors [4] will also be attributable to the MPFC function. A well-functioning MPFC may mediate the association between perceived happiness and health and well-being.

Happiness is a positive feeling characterized by contentment, satisfaction, joy, pleasure, or love. Dopamine plays an important role in the expression of positive emotions [10-12]. The dopaminergic network within the brain, which projects to the prefrontal cortex and striatum from the midbrain region, is known as the "brain reward system" [10,11], and our results indicated that the reward-related brain regions including the dorsal striatum were strongly activated in individuals with high levels of perceived happiness. Findings have suggested that dopaminergic function deficiency may be a possible explanation for depression [13] and that antidepressant treatment facilitated dopamine release in the nucleus accumbens of a genetic animal model of depression [14]. Furthermore, activation of reward-related brain regions can improve physiological functions including immune functions [7]. Thus, brain dopaminergic function may be a key factor in the positive effects of perceived happiness levels.

The present study also indicated that activation of the insula and SMA was higher in the high happiness group than in the low happiness group when participants looked at a favorite person. The insular cortex is involved in the perception of signals arising from the body and plays an important role in emotional experience [15-17]. We have recently shown that parasympathetic activity increases after an individual experiences positive emotions and that activity of the insular cortex is associated with changes in peripheral autonomic activity [18]. Although the present study could not measure the peripheral autonomic activity during the task because of technical limitations, it is possible that parasympathetic activity was greater in the high happiness group than in the low happiness group when positive emotions were elicited, and the greater insula activity in the high happiness group reflected this. Moreover, one study indicated a positive correlation between SMA activity and laughter/smiling; therefore, SMA activity reflects motor activity associated with these actions [19]. The individuals with high levels of happiness probably smiled often while watching the positive film compared with those having low levels of happiness. Therefore, positive emotion-related brain regions may have been highly stimulated upon sight of a favorite person by participants of the high happiness group compared with those of the low happiness group.

Certain limitations of this study must be recognized. Some reports have noted gender-related differences with regard to perceived happiness level and physiological reactivity $[5,20]$, and we did not investigate the effect of gender. Thus, the generalizability of the present findings must be further tested using a larger sample size that includes both male and female participants.

\section{CONCLUSIONS}

The findings of this study indicate that perceived happiness level is associated with evocation of positive emotions via MPFC function. High MPFC function in individuals with high perceived happiness levels may enhance psychological and physiological wellness.

\section{ACKNOWLEDGEMENTS}

This work was supported by Grant-in-Aid for Young Scientists (B) from the Japan Society for the Promotion of Science (22700683 to $\mathrm{MM})$.

\section{REFERENCES}

[1] Magalhaes, A.C., Holmes, K.D. and Dale, L.B., CompsAgrar, L., Lee, D. and Yadav, P.N. et al. (2010) CRF receptor 1 regulates anxiety behavior via sensitization of 5-HT2 receptor signaling. Nat Neuroscience, 13, 622-629. doi:10.1038/nn.2529

[2] Maisel, A.S., Knowlton, K.U., Fowler, P., Rearden, A., Ziegler, M.G. and Motulsky, H.J. et al. (1990) Adrenergic control of circulating lymphocyte subpopulations. Effects of congestive heart failure, dynamic exercise and terbutaline treatment, The Journal of Clinical Investigation. 85, 462-467. doi:10.1172/JCI114460

[3] Jacobs, J.R. and Bovasso, G.B. (2000) Early and chronic stress and their relation to breast cancer. Psychological Medicine, 30, 669-678. doi:10.1017/S0033291799002020

[4] Horiuchi, S., Tsuda, A., Hashimoto, E., Kai, H. and Wenjie, H. (2008) Effect of perceived happiness level on cardiac response to mental stress testing: a pilot study. $J a-$ pan Journal Biofeedback Research, 35, 93-98.

[5] Shimai, S., Otake, K., Utsuki, N., Ikemi, A. and Lyubomirsky, S. (2004) Development of a Japanese version of the subjective happiness scale (SHS) and examination of its validity and reliability. Japan Journal Public Health, 51, 845-853.

[6] Juckel, G., Mendlin, A. and Jacobs, B.L. (1999) Electrical stimulation of rat medial prefrontal cortex enhances forebrain serotonin output: implications for electroconvulsive therapy and transcranial magnetic stimulation in depression. Neuropsychopharmacology, 21, 391-398. doi:10.1016/S0893-133X(98)00097-9

[7] Matsunaga, M., Isowa, T., Kimura, K., Miyakoshi, M., Kanayama, N. and Murakami, H. et al. (2008) Associations among central nervous, endocrine, and immune activities when positive emotions are elicited by looking at 
a favorite person. Brain Behavior and Immunity, 22, 408-417.doi:10.1016/j.bbi.2007.09.008

[8] Friston, K.J., Holmes, A.P., Worsley, K.J., Poline, J.-P., Frith, C.D. and Frackowiak, R.S.J. (1995) Statistical parametric maps in functional imaging: a general linear approach. Human Brain Mapping, 2, 189-210. doi: 10.1002/hbm.460020402

[9] Ohira, H., Isowa, T., Nomura, M., Ichikawa, N., Kimura, K. and Miyakoshi, M. et al. (2008) Imaging brain and immune association accompanying cognitive appraisal of an acute stressor. Neuroimage, 39, 500-514. doi:10.1016/j.neuroimage.2007.08.017

[10] Aron, A., Fisher, H., Mashek, D.J., Strong, G., Li, H. and Brown, L.L. (2005) Reward, motivation, and emotion systems associated with early-stage intense romantic love. Journal of Neurophysiology, 94, 327-337. doi:10.1152/jn.00838.2004

[11] Bartels, A. and Zeki, S. (2004) The neural correlates of maternal and romantic love. NeuroImage, 21, 1155-1166. doi:10.1016/j.neuroimage.2003.11.003

[12] Verhoeff, N.P., Christensen, B.K., Hussey, D., Lee, M., Papatheodorou, G. and Kopala, L. et al. (2003) Effects of catecholamine depletion on D2 receptor binding, mood, and attentiveness in humans: a replication study. Pharmacology Biochemistry Behavior, 74, 425-432. doi:10.1016/S0091-3057(02)01028-6

[13] Yadid, G. and Friedman, A. (2008) Dynamics of the dopaminergic system as a key component to the understanding of depression. Progress in Brain Research, 172 , 265-286. doi:10.1016/S0079-6123(08)00913-8

[14] Roth-Deri, I., Friedman, A., Abraham, L., Lax, E., Flaumenhaft, Y. and Dikshtein, Y. et al. (2009) Antidepressant treatment facilitates dopamine release and drug seeking behavior in a genetic animal model of depression. Europe Journal of Neuroscience, 30, 485-492. doi:10.1111/j.1460-9568.2009.06840.x

[15] Critchley, H.D., Mathias, C.J., Josephs, O., O’Doherty, J., Zanini, S. and Dewar, B.K. et al. (2003) Human cingulate cortex and autonomic control: converging neuroimaging and clinical evidence. Brain, 126, 2139-2152. doi:10.1093/brain/awg216

[16] Critchley, H.D., Wiens, S., Rotshtein, P., Ohman, A. and Dolan, R.J. (2004) Neural systems supporting interoceptive awareness, Nature Neuroscience, 7, 189-195. doi:10.1038/nn1176

[17] Bechara, A. and Naqvi, N. (2004) Listening to your heart: interoceptive awareness as a gateway to feeling. News and views, 7, 102-103. doi:10.1038/nn0204-102

[18] Matsunaga, M., Isowa, T., Kimura, K., Miyakoshi, M., Kanayama, N. and Murakami, H. et al. (2009) Associations among positive mood, brain, and cardiovascular activities in an affectively positive situation. Brain Research, 1263, 93-103. doi:10.1016/j.brainres.2009.01.027

[19] Iwase, M., Ouchi, Y., Okada, H., Yokoyama, C., Nobezawa, S. and Yoshikawa, E. et al. (2002) Neural substrates of human facial expression of pleasant emotion induced by comic films: a PET study. Neuroimage, 17, 758-768. doi:10.1006/nimg.2002.1225

[20] Bosch, J.A., Berntson, G.G., Cacioppo, J.T. and Marucha, P.T. (2005) Differential mobilization of functionally distinct natural killer subsets during acute psychological stress. Psychosomatic Medicine, 67, 366-375. doi:10.1097/01.psy.0000160469.00312.8e 